\title{
Genetic diversity and thermal performance in invasive and native populations of African fig flies
}

Comeault, Aaron; Wang, Jeremy; Tittes, Silas; Isbell, Kristin; Ingley, Spencer; Hurlbert, Allen; Matute, Daniel

\section{Molecular Biology and Evolution}

Published: 28/02/2020

Publisher's PDF, also known as Version of record

Cyswllt i'r cyhoeddiad / Link to publication

Dyfyniad o'r fersiwn a gyhoeddwyd / Citation for published version (APA):

Comeault, A., Wang, J., Tittes, S., Isbell, K., Ingley, S., Hurlbert, A., \& Matute, D. (2020). Genetic diversity and thermal performance in invasive and native populations of African fig flies. Molecular Biology and Evolution. https://doi.org/10.1093/molbev/msaa050

\footnotetext{
Hawliau Cyffredinol / General rights

Copyright and moral rights for the publications made accessible in the public portal are retained by the authors and/or other copyright owners and it is a condition of accessing publications that users recognise and abide by the legal requirements associated with these rights.

- Users may download and print one copy of any publication from the public portal for the purpose of private study or research.

- You may not further distribute the material or use it for any profit-making activity or commercial gain

- You may freely distribute the URL identifying the publication in the public portal?
}

Take down policy

If you believe that this document breaches copyright please contact us providing details, and we will remove access to the work immediately and investigate your claim. 
Article; Discoveries

Genetic diversity and thermal performance in invasive and native populations of African fig flies

Aaron A. Comeault ${ }^{1}$, Jeremy Wang $^{2}$, Silas Tittes $^{3}$, Kristin Isbell ${ }^{4}$, Spencer Ingley ${ }^{5}$, Allen H. Hurlbert $^{2}$, Daniel R. Matute ${ }^{2}$

${ }^{1}$ School of Natural Sciences, Bangor University, Bangor, Gwynedd, LL57 2DGA, UK

${ }^{2}$ Department of Genetics, University of North Carolina, Chapel Hill, NC, 27599, USA

${ }^{3}$ Department of Evolution and Ecology, University of California, Davis, CA, 95616, USA

${ }^{4}$ Department of Biology, University of North Carolina, Chapel Hill, NC, 27599, USA

${ }^{5}$ Faculty of Sciences, Brigham Young University, Hawaii, Laie, 96762 USA

Corresponding author: a.comeault@bangor.ac.uk

Author contributions: Conceptualization: AAC, AHH, DRM; Data Curation: AAC, JW, ST, KI, SI; Formal Analysis: AAC, JW, ST; Funding Acquisition: AHH, DRM; Investigation: AAC, JW, ST; Supervision: AHH, DRM; Visualization: AAC, ST; Writing: AAC wrote the original draft with subsequent input from all authors.

Data availability: Raw sequences at the NCBI short read archive under BioProject PRJNA604690; genome assemblies, annotations, and phenotypic data at Dryad DOI: https://doi.org/10.5061/dryad.866t1g1n3.

(c) The Author(s) 2020. Published by Oxford University Press on behalf of the Society for Molecular Biology and Evolution. This is an Open Access article distributed under the terms of the Creative Commons Attribution License (http://creativecommons.org/licenses/by/4.0/), which permits unrestricted reuse, distribution, and reproduction in any medium, provided the original work is properly cited. 


\section{Abstract}

During biological invasions, invasive populations can suffer losses of genetic diversity that are predicted to negatively impact their fitness/performance. Despite examples of invasive populations harboring lower diversity than conspecific populations in their native range, few studies have linked this lower diversity to a decrease in fitness. Using genome sequences, we show that invasive populations of the African fig fly, Zaprionus indianus, have less genetic diversity than conspecific populations in their native range and that diversity is proportionally lower in regions of the genome experiencing low recombination rates. This result suggests that selection may have played a role in lowering diversity in the invasive populations. We next use interspecific comparisons to show that genetic diversity remains relatively high in invasive populations of $Z$. indianus when compared to other closely related species. By comparing genetic diversity in orthologous gene regions, we also show that the genome-wide landscape of genetic diversity differs between invasive and native populations of $Z$. indianus, indicating that invasion not only affects amounts of genetic diversity, but also how that diversity is distributed across the genome. Finally, we use parameter estimates from thermal performance curves measured for 13 species of Zaprionus to show that $Z$. indianus has the broadest thermal niche of measured species, and that performance does not differ between invasive and native populations. These results illustrate how aspects of genetic diversity in invasive species can be decoupled from measures of fitness, and that a broad thermal niche may have helped facilitate $Z$. indianus's range expansion.

\section{Introduction}

Populations of invasive species can experience extreme demographic histories. For example, processes such as bottlenecks, inbreeding, hybridization, and multiple introductions can all operate in populations of invasive species (Ellstrand and Schierenbeck 2000; Kolbe et al. 2004; Dlugosch and Parker 2008; Hovick and Whitney 2014; Barker et al. 2019). More generally, the impact of demography on levels of genetic variation in invasive populations has been a major focus in the field of invasion genetics since its inception more than 60 years ago (Baker and Stebbins 1965; Lee 2002; Dlugosch et al. 2015); especially with respect to its effect on invasive species' ability to adapt to novel environments. Under the assumption that invasive species experience strong bottlenecks, and in some cases inbreeding, an erosion of genetic diversity is predicted to reduce fitness and impose constraints on a population's ability to persist in and adapt to novel environments (Markert et al. 2010; Agashe et al. 2011; Agashe and Bolnick 2010). The 
fact that invasive species are frequently able to successfully colonize and adapt to novel environments, despite the negative consequences that a loss of diversity is expected to have on fitness (and by extrapolation, population growth rates), has led to the idea of the "genetic paradox of invasive species" (Allendorf and Lundquist 2003; Estoup et al. 2016).

While studies have shown that some invasive populations experience a loss of genetic diversity relative to populations in the species' native range (Tsutsui et al. 2000; Grapputo et al. 2005; Michaelides et al. 2018), these findings are frequently based on a small number of putatively neutral genetic markers. Others have shown that invasive populations can maintain high levels of genetic diversity through processes such as hybridization and multiple introductions stemming from different source populations in the species' native range (Stepien et al. 2005; Lavergne and Molofsky 2007; Facon et al. 2008). The paradoxical nature of invasions has therefore been called into question (Dlugosch et al. 2015; Estoup et al. 2016).

In cases where invasive populations show less genetic diversity than native populations, at least two general arguments have been made against the idea of a genetic paradox in the invasive populations. First, lower genetic diversity at neutral loci is not equivalent to a loss of adaptive genetic variation: invasive populations may show reduced genetic variation at neutral loci but retain variation at loci that are important for maintaining fitness and adapting to novel environments (Dlugosch et al. 2015; Estoup et al. 2016). Second, invasive populations may not need to adapt to the habitats they are colonizing, therefore removing the paradox altogether (Estoup et al. 2016). For example, populations may adapt to human altered or disturbed environments in their native range, thereby facilitating subsequent range expansions into "anthropogenic" environments (a process termed "anthropogenically induced adaptation to invade": (Hufbauer et al. 2012)).

Theory predicts that changes in population size will affect both the overall amount and also the type of genetic variation found within a population. Genetic drift in small populations can, for example, lead to the fixation of weakly deleterious mutations segregating at low frequencies in source populations (Gillespie 1994; Marsden et al. 2016; Rogers and Slatkin 2017), and populations that are either increasing (e.g. a growing invasive population) or decreasing (e.g. bottlenecked populations at the front of a range expansion) in size are expected to fix novel beneficial or deleterious mutations, respectively, with higher probability than populations of constant size (Otto and Whitlock 1997). These theoretical expectations have important 
implications for the dynamics of adaptation (and maladaptation) during range expansions. We therefore require a better understanding of how biological invasions and range expansions affect genome-wide patterns of diversity and how these changes may alter the average fitness of individuals in invading populations.

When explicit links between genetic variation and adaptive phenotypic variation have not been made, a comparative approach can be used to gain insight into general effects that invasion has on genetic variation. First, genome-wide data can be used to gain a more nuanced understanding of patterns of genetic variation within populations found in invasive versus native parts of a species' range. For example, genetic diversity varies across the genome (Langley et al. 2012; Dutoit et al. 2017) and genomic data from invasive and native populations could be used to test whether genetic diversity is consistently reduced across the genome. This approach has been used to suggest that the interaction between recombination rate, gene structure, and selection can generate conserved patterns of variation in genetic diversity across the genomes of different species (Langley et al. 2012; Dutoit et al. 2017). However, we do not know how invasion may alter genetic diversity at a genomic scale. Interspecific comparisons can therefore be used to generate a better understanding of if/how invasion affects the genome-wide distribution of genetic diversity.

Second, while studies of genetic variation in invasive species tend to focus on comparisons between invasive and native populations, comparisons between other invasive species and closely related non-invasive species can reveal whether the reduction in genetic diversity observed in an invasive population results in levels of genetic diversity that are below broadly observed levels. This type of comparison can be informative if populations that show comparable genetic diversity to the invasive population of interest also show evidence of contemporary adaptation to different environments. However, as mentioned above, summary statistics of genome-wide trends in genetic diversity may not reflect amounts of additive-genetic variation, and we require additional information to understand the effects of invasion on performance and adaptation in invasive populations.

From a phenotypic perspective, quantifying performance across different environments can be used to assess whether range expansions associated with biological invasions have affected the fitness of individuals in invasive populations. If inbreeding and small population size had led to a decrease in fitness, one prediction is that between-population crosses will display higher fitness 
than crosses carried out within an inbred population (i.e. heterosis (Oakley et al. 2019)). More generally, if invasive populations suffer from inbreeding depression, we would predict that they will display lower fitness (or some measure correlated with fitness) relative to outbred populations that are found in the native portion of the species' range (Oakley et al. 2019). Therefore, by combining genome-wide surveys of genetic variation with phenotypic measurements of performance, we can gain a better understanding of the processes affecting genetic variation during biological invasions and how those processes might affect the fitness of individuals in invading populations.

Here, we analyze whole genome sequences collected from 93 individuals sampled across 7 species and 16 populations of African fig fly (genus Zaprionus; Figure 1) to test whether invasive populations of $Z$. indianus are outliers with respect to the genetic variation they harbor. We also use these species to quantify the genome-wide diversity landscape and test whether it has been altered in invasive populations of $Z$. indianus. Finally, we estimate thermal performance curves for 21 populations across 13 species of African fig fly to test whether there is any general relationship between levels of genetic variation and performance across a broad range of temperatures. We find that invasive populations of $Z$. indianus have lower genetic diversity than populations in their native range, and that both the presence or absence of genes and local recombination rates affect amounts of genetic diversity. However, genetic diversity in invasive populations tends to remain as high or higher than in non-invasive species of Zaprionus. Estimates of thermal performance curves indicate that $Z$. indianus has the broadest thermal niche of the species we tested, and despite lower genetic diversity, invasive populations of $Z$. indianus do not show reduced performance relative to populations in their native range. These results suggest that a broad thermal niche may have facilitated the range expansion of $Z$. indianus. In turn, large populations (and high genetic diversity) in Z. indianus's native range may have acted to buffer invasive populations against critical losses of genetic diversity. 
a
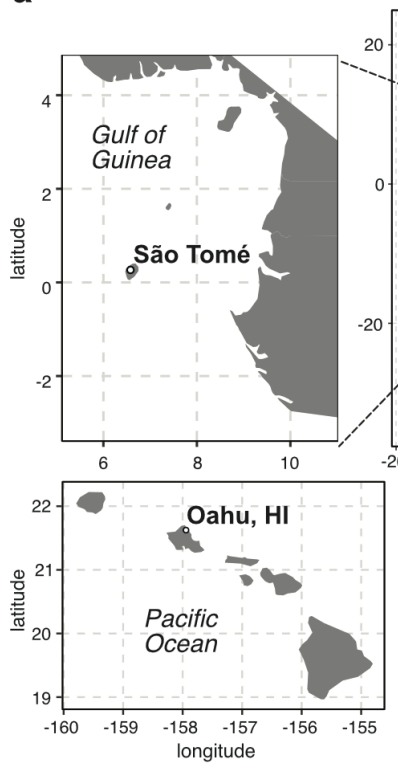
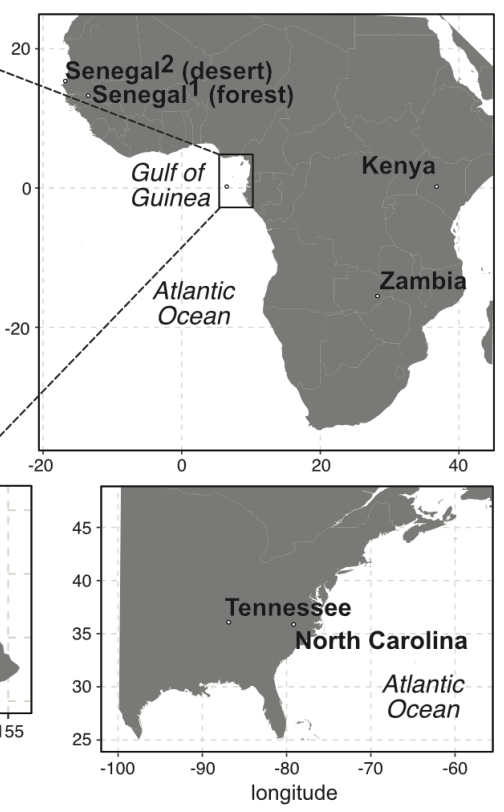

b

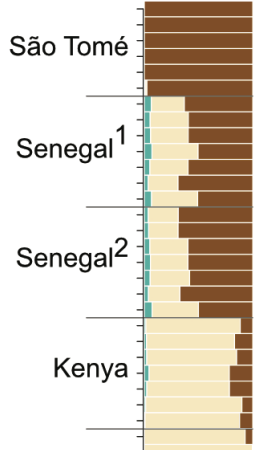

Zambia

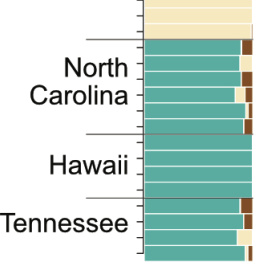

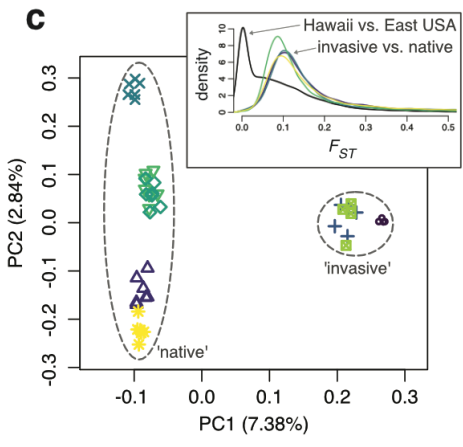

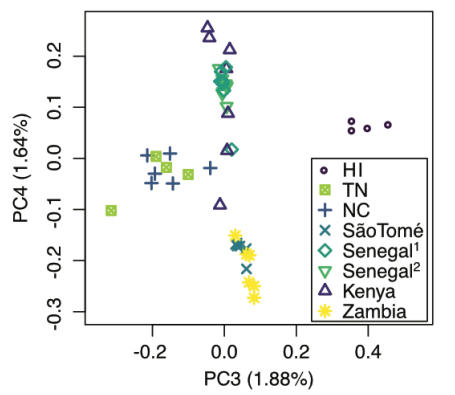

Figure 1. Collection locations across sub-Saharan Africa, eastern North America, and Hawaii (a) and genetic differentiation among $Z$. indianus samples (b and c). (a) All collection locations are highlighted with bold type. Zaprionus indianus was sampled from all locations, $Z$. tuberculatus was sampled from São Tomé and Senegal (forest site), Z. africanus from São Tomé and Kenya, and Z. inermis, Z. tsacasi, Z. taronus, and Z. nigranus from São Tomé. Invasive populations of $Z$. indianus are differentiated from populations in their native range (b and $\mathbf{c}$ ), with the strongest genetic differentiation between invasive and native populations (c; PC1) and among African populations (c; PC2). Invasive populations in the eastern USA and Hawaii also show weak differentiation (c; PC3). The inset in the top right of panel c shows the genome-wide distribution of differentiation ( $F_{S T} ; 5 \mathrm{~kb}$ genomic windows) between invasive $Z$. indianus and each of the four African populations (Senegal sample locations grouped as one population), and also between eastern USA and Hawaiian samples (black line).

\section{Results}

We estimated genetic differentiation among populations of $Z$. indianus to test whether genetic affinities between invasive and native populations suggested potential sources of the invasion (Figure 1; Supplementary Materials online). Population assignment (Figure 1b), principal component analysis (Figure 1c), and genome-wide estimates of differentiation (FST) showed that invasive populations were closely related to one another and genetically differentiated from African populations. Despite broad geographic sampling in Africa, these analyses did not indicate that invasive populations were more closely related to any one African population (median $\mathrm{F}_{\mathrm{ST}}$ ranged from 0.14 to 0.19 between invasive and African populations), as would be expected if they were recently derived from that population, or an unsampled, but closely related one. Below we 
focus on aspects of genetic diversity within each population rather than differentiation among them.

Invasive populations of Z. indianus have less genetic diversity than native populations

A central tenet of the genetic paradox of invasive species is that relatively few individuals colonize invasive parts of their range and that these populations are subject to a loss of genetic diversity, and potentially to inbreeding (Allendorf and Lundquist 2003; Estoup et al. 2016). We tested for relatedness and inbreeding in our samples, but found no evidence that any of the individuals we sampled were closely related (all kinship coefficients estimated within populations $<0.017$ ). There was also no evidence for inbreeding in the invasive range of $Z$. indianus relative to its native range $\left(F_{1,17}=2.37 ; P=0.14\right.$; Figure $\left.S 1\right)$. Below we therefore focus on levels of genetic diversity segregating within populations.

We computed nucleotide diversity $(\pi)$, the number of segregating sites $(S)$, and Tajima's $D$ in nonoverlapping $5,000 \mathrm{bp}$ windows across the genome for 16 populations of 7 species of Zaprionus. Among populations of the invasive $Z$. indianus, populations sampled in the invasive part of the species' range display significantly less genetic diversity than populations sampled in their native range: the median number of segregating sites $(S)$ in $5 \mathrm{~kb}$ genomic windows was between 120 and 160 across North American and Hawaiian populations (5\% empirical quantiles: 0 to 10 SNPs; Figure 2; Table S4) and 206 to 233 across African populations (5\% empirical quantiles: 21 to 37 SNPs; Figure 2; Table S4; Supplementary Materials online). We observed similarly low genetic diversity in invasive, relative to native, populations of $Z$. indianus when we restricted our analysis to genomic windows that overlap an annotated BUSCO gene (Figure S2; Table S5; Supplementary Materials online). These results are in line with studies in other systems that have shown lower genetic diversity in invasive populations than in native populations of invasive species (Grapputo et al. 2005; Michaelides et al. 2018).

Tajima's $D$ also varied across populations of $Z$. indianus, with invasive populations showing positive median genome-wide estimates of Tajima's $D(0.13$ to 0.5$)$, consistent with a recent contraction in population size, and native populations showing negative median estimates of Tajima's $D$ (-0.48 to -0.72), consistent with range expansion (Figure 2; Table S4; Supplementary Materials online). A positive genome-wide estimate of Tajima's $D$ in the invasive populations of $Z$. indianus, together with lower genetic diversity relative to the native populations, points to an 
active loss of lost genetic variation occurring in the invasive populations during their range expansion. However, because the ancestral population that gave rise to the invasive populations is unknown, additional population samples and future demographic analyses are required to test the specific demographic scenarios driving the patterns we observe with respect to genetic diversity in these populations.

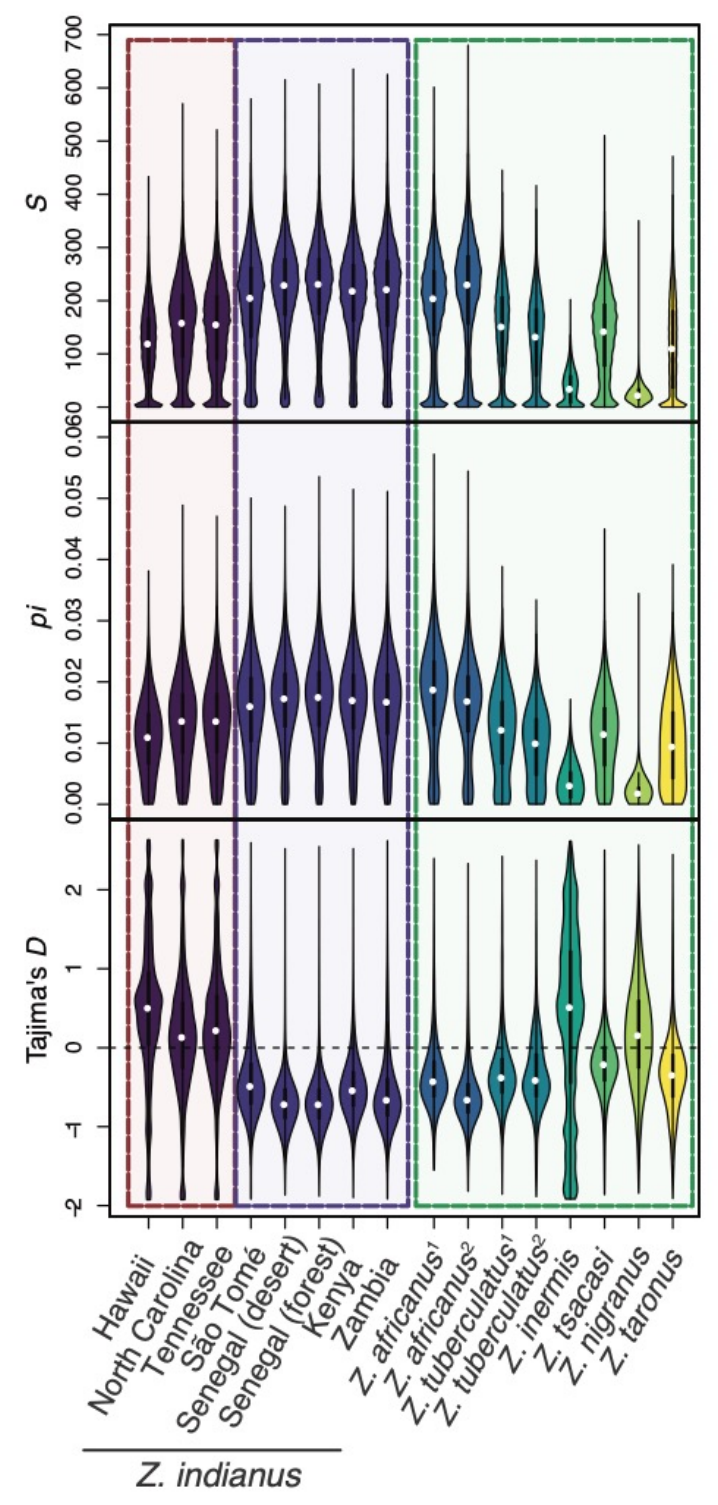

Figure 2. Estimates of genetic diversity summarized across $5 \mathrm{~kb}$ genomic windows for each population included in this study. Colored backgrounds group populations as invasive $Z$. indianus (three leftmost violins), native $Z$. indianus (five central violins), and other species (eight rightmost violins). See Figure S2 for estimates for windows overlapping with BUSCO annotations and Table S4 for estimates in all subsamples from populations where we sampled more than four individuals. ${ }^{1}$ : population of $Z$. africanus or $Z$. tuberculatus sampled from Sao Tome; ${ }^{2}$ : population of $Z$. africanus from Kenya or $Z$. tuberculatus from Senegal (forest site).

Genetic diversity in invasive populations of $\mathbf{Z}$. indianus is not exceptionally low

While levels of genetic diversity were reduced in invasive populations of $Z$. indianus, we found that these populations still harbor as much, or more, genetic diversity than naturally occurring noninvasive species of Zaprionus (Figure 2). For example, $S$ within populations of $Z$. tuberculatus and Z. tsacasi (median $S=141$ and 143 , respectively) is comparable to $S$ in invasive populations of $Z$. indianus (median $S=120$ to 140 ). By contrast, populations of Z. nigranus, Z. taronus, and Z. inermis all show markedly less genetic diversity (median $S=23,106$, and 35 , respectively) than any of the $Z$. indianus populations we sampled. The broadly distributed species $Z$. africanus (the closest relative to $Z$. indianus included in this study) showed amounts of genetic diversity comparable to those in native populations of $Z$. indianus. It is also worth noting that the level of genetic diversity we observe in invasive and native 
populations of $Z$. indianus is similar to the distantly related invasive Drosophilid fly $D$. suzukii (nucleotide diversity $=0.88 \%-2.28 \%$; (Adrion et al. 2014)), but somewhat greater than estimates for $D$. melanogaster (nucleotide diversity across multiple studies and populations ranges from 0.1\% to 0.9; (Begun and Aquadro 1992; Langley et al. 2012; Pool et al. 2012)).

Similar to comparisons among populations of $Z$. indianus, we recovered the same relationship when restricting our analysis to genomic windows that overlap an annotated BUSCO gene (Tables S4 and S5). Patterns of genetic diversity across populations of $Z$. indianus and other species of Zaprionus suggest that, under the assumption that the amount of genetic diversity in non-invasive species is a reasonable proxy for the amount of genetic diversity a population can have without negative fitness effects, high levels of genetic diversity in the native range of invasive species may buffer invasive populations against losses of diversity below levels that would negatively affect fitness or adaptive potential.

\section{Genetic diversity is affected by genome architecture}

While population bottlenecks occurring in invasive populations are expected to lower genetic diversity broadly across the genome (Hyten et al. 2006; Ellegren and Galtier 2016), other processes can act locally within the genome and either reduce (e.g. selective sweeps; (Charlesworth et al. 1997)) or maintain genetic diversity (e.g. balancing selection; (Aguilar et al. 2004; Charlesworth 2006; Lindtke et al. 2017)). The fitness consequences of diversity across loci is also not expected to be equal. Genetic diversity therefore varies greatly across the genome and the interaction between selection and recombination rate can lead to a genome-wide "landscape" of genetic diversity that is correlated with aspects of genome architecture such as recombination rates or gene density (Begun and Aquadro 1992; Nachman 2001; Burri et al. 2015; Dutoit et al. 2017). We generated gene annotations and used estimates of recombination rates to test the relationship between genetic diversity in invasive and native populations of $Z$. indianus and these features of the genome.

Across all genomic windows, we found that the amount of genetic diversity $(S)$ within a given genomic window varied depending on whether that window overlapped-with, was adjacent-to, or was distant-from an annotated gene (population level comparisons: GLMs: all $P<0.0001$ ). Genetic diversity tended to be lower in windows that overlap an annotated gene compared to windows that were either within or outside of $5 \mathrm{~kb}$ from an annotated gene (12 of 15 populations; 
binomial test: $P=0.035$; Figure S3; Supplementary Materials online). Three populations did not follow this trend: the two Z. africanus populations and the Z. tuberculatus population sampled from Senegal. For populations that had less diversity in windows that overlapped an annotated gene, mean diversity tended to be 3.5 to $21.5 \%$ lower than in windows within $5 \mathrm{~kb}$ of an annotated gene, and 0.6 to $32.9 \%$ lower than in windows further than $5 \mathrm{~kb}$ from an annotated gene (Table S6). We did not, however, observe an interaction between invasion status (invasive Z. indianus, native $Z$. indianus, or non-invasive Zaprionus) and window-location relative to annotated genes and the median amount of genetic diversity $(S)$ observed across windows (GLM: $P=0.99$ ). The amount of genetic diversity within a genomic region is therefore affected by the presence (or absence) of genes, but broad scale differences in diversity between "genic" and "non-genic" regions is not systematically altered - for example, due to selection preferentially maintaining genetic diversity in or around genes - during the course of invasion.

We next explored the relationship between genetic diversity $(S)$ and mean estimates of population-scaled recombination rates in $5 \mathrm{~kb}$ genomic windows (Materials and Methods). Consistent with previous studies (Begun and Aquadro 1992; Begun et al. 2007; Burri et al. 2015; Dutoit et al. 2017; Samuk et al. 2017), S was positively correlated with recombination rate across genomic windows in all Z. indianus populations (all Spearman's $\rho>0.5$; Figure 3a). The strength of this correlation did not systematically differ between invasive and native populations of $Z$. indianus (linear model: $F_{1,12}=0.1025 ; P=0.7543$ ); however, the mean difference in $S$ between invasive and native populations of $Z$. indianus was correlated with local recombination rate (analysis of windows with mean $S$ between 150 and 300 in $Z$. indianus's native range; Figure 3b). This correlation was weak when analyzing the raw difference in $S$ (Spearman's $\rho=0.071$; Figure S4; Supplementary Materials online), but was modest when the difference in $S$ was scaled by mean $S$ for genomic windows binned by recombination rate quantiles (Spearman's $\rho=0.231$; Figure $3 b$ ). This finding shows that during invasions, the loss of diversity is not uniform across the genome, but is greater in genomic regions experiencing lower recombination rates. 
a

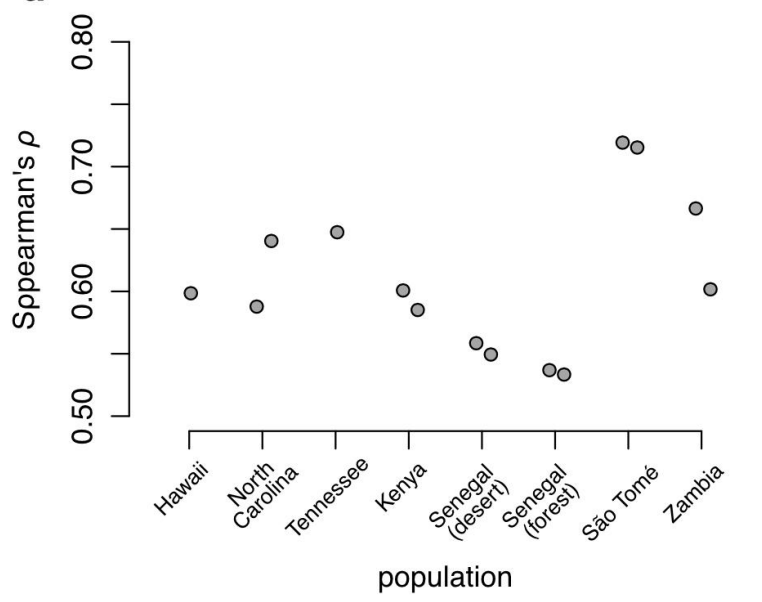

b

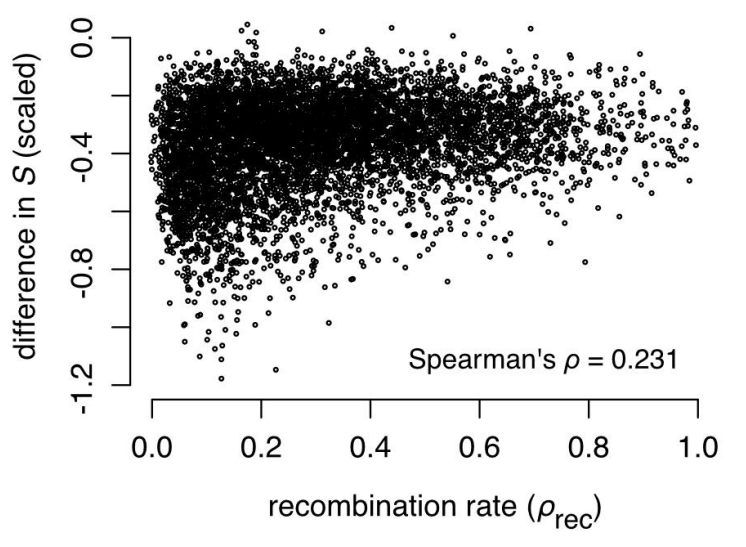

Figure 3. Correlations between genetic diversity (the number of segregating sites: $S$ ) and recombination rate across populations of $Z$. indianus (a). Correlations did not systematically differ between populations in the invasive and native regions of the species' range. However, the mean difference in diversity for a given genomic window was positively correlated with recombination rate (b). Populations with two points in panel a represent populations where we sampled more than 4 individuals and estimated $S$ using two independent random subsamples of those individuals. In panel $\mathbf{b}$, the difference in $S$ (mean in invasive populations - mean in native populations) was scaled by mean levels of diversity for a given recombination rate quantile.

Invasion alters the genome-wide "landscape" of genetic diversity

As a second test of whether the genome-wide distribution of diversity is altered during biological invasions, we compared genetic diversity between species in genomic windows that spanned annotated BUSCO genes. Consistent with a genome-wide landscape of genetic diversity, we found that genetic diversity $(S)$ within annotated BUSCO genes was correlated between populations and species of Zaprionus (Figure 4; see Figure S5 for randomization test; Supplementary Materials online). As expected, correlations in $S$ were weaker for interspecific comparisons than for intraspecific comparisons (Figure 4a). The strongest interspecific correlation we observed was between Z. indianus from Zambia and Z. africanus from São Tomé (Spearman's $\rho=0.4504$; Figure $4 \mathrm{~b}$ ) and the weakest was between $Z$. inermis from São Tomé and $Z$. africanus from Kenya (Spearman's $\rho=0.1888$; Figure 4c). Across all pairwise comparisons, there was a significant negative relationship between the correlation in $S$ across the genome and the genetic distance between the species being tested (Mantel $r=-0.816 ; P=0.0001$; Figures $5 \mathrm{a}$ and $\mathrm{b}$; pairwise sequence differences ranged from 4.3 to $13.7 \%$ for the species compared here; see Table S7; Supplementary Materials online). This trend was even stronger among comparisons between more closely related species pairs (i.e. "within-clade" comparisons; Mantel $r=-0.83$; 
Figure 5c). The pattern of weaker correlations in $S$ between more genetically diverged species is consistent with evolution of aspects of genome architecture (e.g. intron size, recommendation rates, and / or transposable element evolution) altering the genome-wide landscape of diversity; however, explicit tests of these mechanisms are still needed.

As with interspecific comparisons restricted to BUSCO windows, genetic diversity $(S)$ was significantly correlated across all genomic windows between populations of $Z$. indianus (Figure $4 d)$. Among African populations of $Z$. indianus, this was not a function of how closely related two populations were: geographically distant populations in Senegal and Kenya or Senegal and Zambia (Figure 1) show among the strongest correlations in $S$ (Spearman's $\rho>0.885$; Figures $4 d \& 4 e)$. In general, the correlation in $S$ was lower when a native and an invasive population were being compared versus comparisons made among native populations (maximum $\rho=0.865$, minimum $\rho=0.694$; Figure 4d \& 4f). Among invasive populations, North Carolina and Tennessee populations showed the strongest correlation in $S$ (Spearman's $\rho>0.878$; Figure $4 \mathrm{~g}$ ); however, this correlation was lower than the five strongest correlations, all observed between African populations.
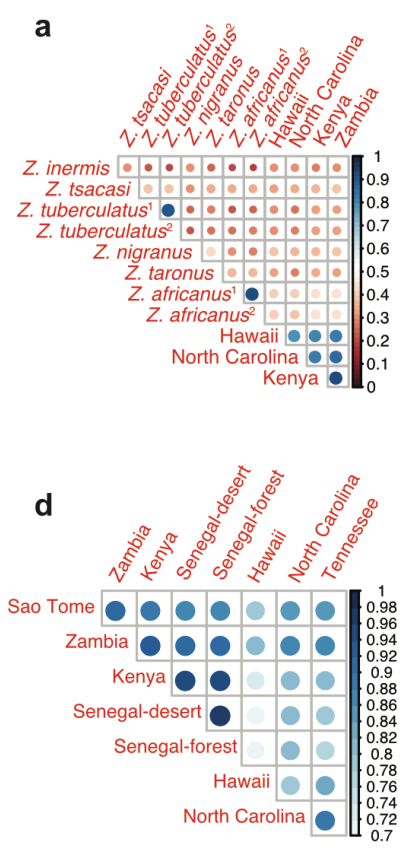

b
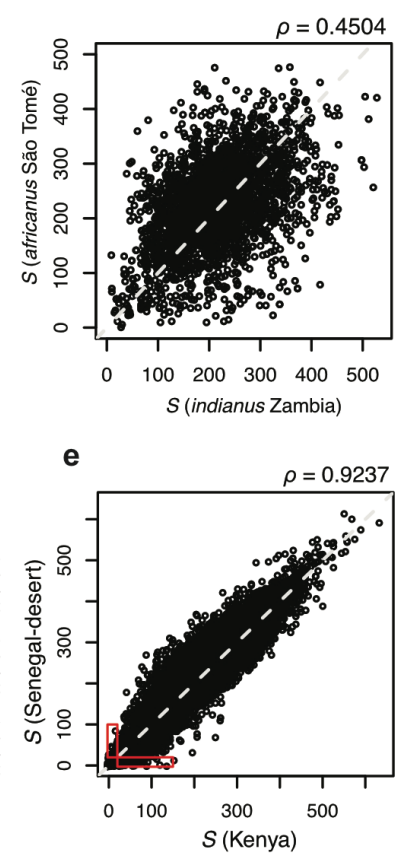

c
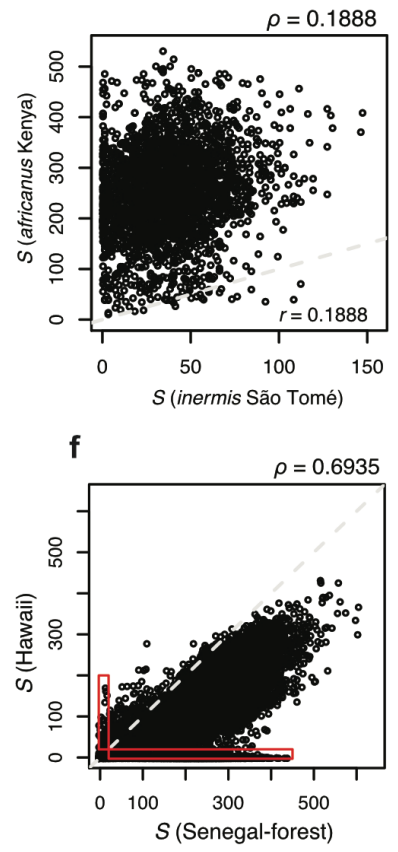

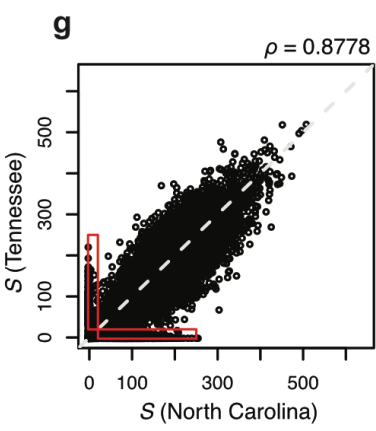

Figure 4. Genetic diversity $(S)$ is correlated across regions of the genome containing annotated single-copy orthologs in interspecific comparisons (a). The closely related species $Z$. africanus from São Tomé and Z. indianus from Zambia had the strongest interspecific correlation in $S$ (b; 
see Figure $4 a$ for phylogeny) and the distantly related species $Z$. africanus from Kenya and $Z$. inermis from São Tomé had the weakest correlation in $S$ (c). Genetic diversity is strongly correlated in all pairwise comparisons between populations of $Z$. indianus (d). Panels $\mathbf{e}$ and $\mathbf{f}$ show data from the strongest and weakest between-population correlations for $Z$. indianus, and panel $\mathbf{g}$ shows the strongest correlation between invasive populations of $Z$. indianus. Red rectangles in panels e through $\mathbf{g}$ highlight genomic windows that have low diversity in one population (fewer than 10 segregating SNPs), but higher diversity in the other (more than 10 segregating SNPs).

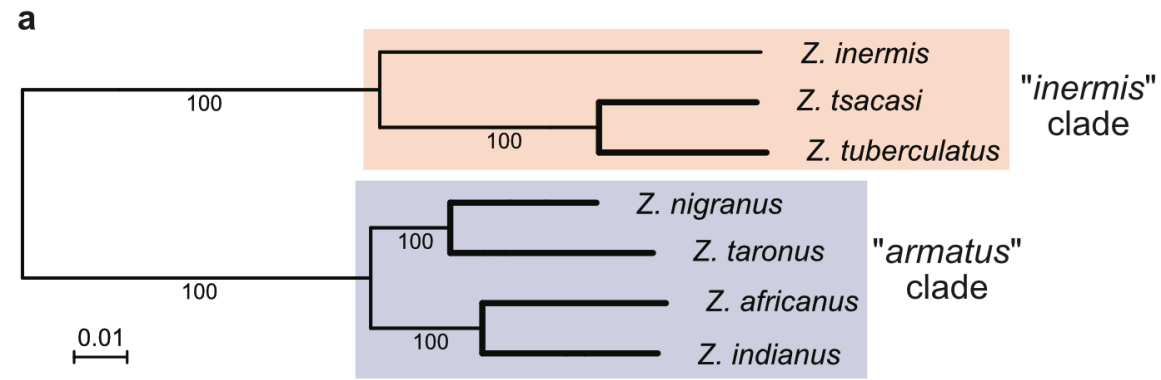

b

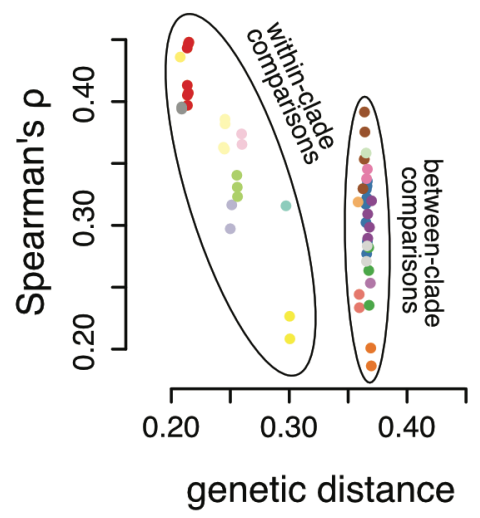

C

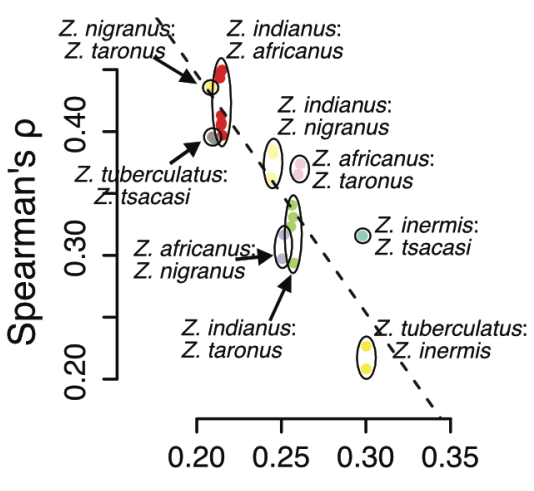

genetic distance

Figure 5. The correlation of genetic diversity $(S)$ across the genomes of different species (a) decreases with increasing genetic distance (b \& c). Maximum likelihood phylogeny in a was estimated with RAxML run on an alignment of 1709 BUSCO genes annotated across all seven species' genomes. Correlation coefficients decreased with increasing genetic distance (Mantel $r$ $=-0.816$; b), with this pattern being particularly evident for within-clade comparisons (highlighted in c).

In contrast to $S$, Tajima's $D$ was either weakly correlated or uncorrelated between species (Figure S5a; Supplementary Materials online), and there was no relationship between the strength of correlation in Tajima's $D$ and phylogenetic distance $(P>0.1)$. By contrast, Tajima's $D$ was significantly correlated across genomic windows between all populations of $Z$. indianus $(P<$ 0.001; Figure S5b; Supplementary Materials online). However, Tajima's $D$ was more weakly 
correlated than $S$ (compare Figure S5b to Figure $4 d$ ). As with $S$, the strongest correlations in Tajima's $D$ were between populations sampled in Africa, consistent with a shared demographic history in the native part of $Z$. indianus' range (Figure S5b and S5c; Supplementary Materials online). By contrast, Tajima's $D$ was only weakly correlated in between-continent comparisons (strongest between-continent $\rho=0.192$; weakest $\rho=0.0167$; Figure S5d; Supplementary Materials online) and moderately correlated between the two populations sampled in the eastern USA (Tennessee and North Carolina; $\rho=0.2002$; Figure S5e; Supplementary Materials online). The fact that Tennessee and North Carolina have both been recently colonized during $Z$. indianus's expansion into North America ( 20 years ago; (Gibert et al. 2016)), yet show relatively modest correlations in Tajima's $D$, illustrates how demographic events can rapidly alter the frequency of alleles across the genome and suggests that these two locations are experiencing semi-independent colonizations, rather than an expansion driven by a single panmictic population.

The correlation of genetic diversity $(S)$ between $Z$. indianus and other species of Zaprionus (Figures 4 and 5) allowed us to test whether this correlation is maintained following biological invasion. We find that interspecific correlations in $S$ are weaker between invasive populations and other species than between native populations and other species (6 of 8 species/population comparisons; Fisher's exact test: $P=0.0093$; Figure 6). When we restrict this same analysis to include one population per species, the correlation in genetic diversity remains weaker between invasive populations of $Z$. indianus and other Zaprionus species than between native populations of Z. indianus and other Zaprionus in four of the 6 species-level comparisons (Fisher's exact test:

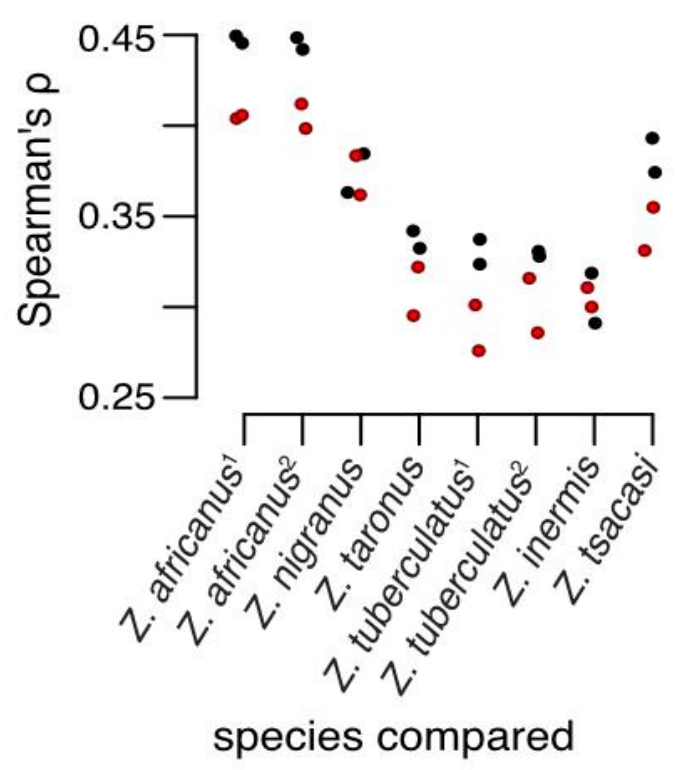
$P=0.03)$. Native populations of $Z$. indianus also never showed consistently weaker correlations than invasive populations (Figure 6). These findings illustrate how demographic and selective processes associated with biological invasions do not only result in a general reduction in diversity in invasive populations, but also alter the genome-wide distribution of that diversity.

Figure 6. The correlation in genetic diversity ( $S$ ) between invasive populations of $Z$. indianus and other species of Zaprionus (dark red points) is 
weaker than the correlation in $S$ between native populations of $Z$. indianus and other species of Zaprionus (black points).

Genetic diversity is correlated with thermal niche breadth

Genetic diversity can, in some cases, be positively correlated with measures of performance or fitness (Briskie and Mackintosh, 2004; Leimu et al., 2006; Markert et al., 2010; Reed and Frankham, 2003). We therefore tested for relationships between genetic diversity and fitness/performance, as measured from thermal performance curves.

We estimated thermal performance curves using a hierarchical Bayesian framework ((Tittes et al. 2019); Supplementary Materials online). Parameters summarizing performance were estimated at the population level, allowing us to make comparisons between populations of $Z$. indianus sampled from three populations from their invasive range in eastern North America (Florida, North Carolina, and New York, USA) and their native range in Africa (São Tomé, Senegal, Kenya, and Zambia).

Among populations of Zaprionus species with estimates of genetic diversity and thermal performance, thermal niche breadth $\left(B_{50}\right)$ and the total area of the thermal performance curve $\left(A_{c}\right)$ were positively associated with genetic diversity (Figure $7 \mathrm{a}$; linear models: $B_{50}: F_{1,11}=10.1 ; P=$ 0.009; $A_{c}: F_{1,11}=7.402 ; P=0.020$; note that $A_{c}$ and maximum fitness parameters were strongly correlated; $r=0.99$ ). By contrast, there was no relationship between levels of genetic diversity and thermal optima $\left(F_{1,11}=1.625, P=0.23\right)$. Linear models fit to our complete data set should however be interpreted with caution because of phylogenetic nonindependence and uneven sampling across species (e.g. we sampled multiple populations of $Z$. indianus, $Z$. africanus, and Z. tuberculatus). We therefore also fit models that only included a single population from each of the seven species sampled from São Tomé (Figure 7b). Using this reduced data set, we still find support for a positive relationship between genetic diversity and thermal niche breadth ( $B_{50}: F_{1,5}$ $\left.=8.244 ; P=0.035 ; R^{2}=0.55\right)$, but no relationship between genetic diversity and $A_{c}$ or thermal optimum (both $P>0.1$ ). These results are consistent with genetic diversity being higher in species that are able to exploit a broad range of thermal environments, potentially due to these species maintaining large population sizes. 
a. all populations

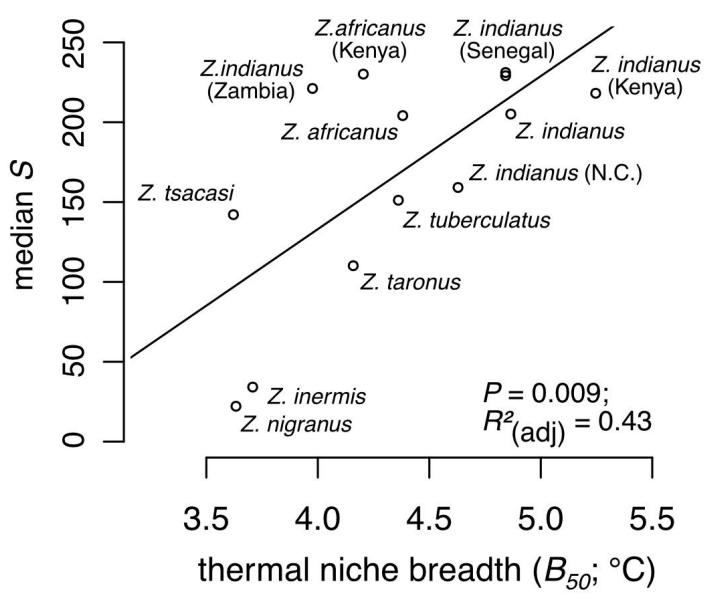

b. São Tomé

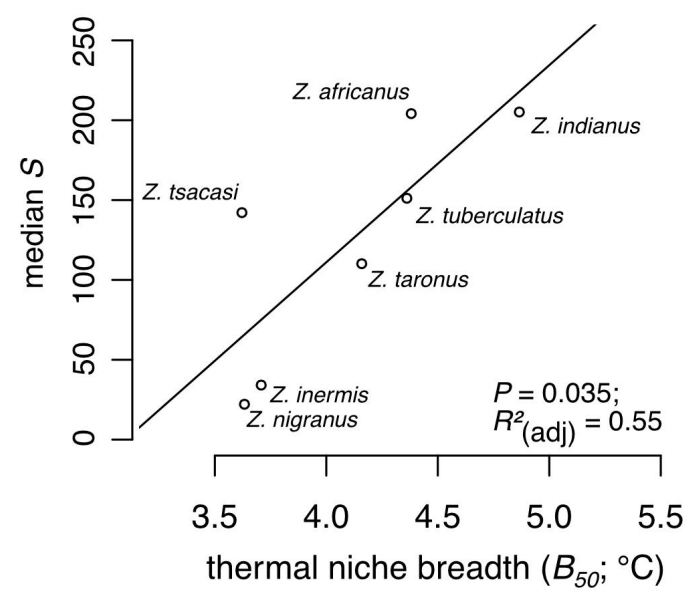

Figure 7. Populations and species with a larger thermal niche breadth $\left(B_{50}\right)$ also harbor more genetic diversity (median number of segregating sites; $S$ ). Results for all populations (a) and for only the seven species sampled on the island of São Tomé (b) are shown. Species names that are not labeled with a location in panel (a) are from São Tomé. Results from linear models testing the relationship between thermal niche breadth and genetic diversity are reported in the bottom right of each panel.

\section{Performance is not reduced in invasive populations}

Among the seven species we collected population genomic data from, $Z$. indianus tended to have the highest estimated $B_{50}$ (Figure 7). We tested the generality of this pattern by estimating thermal performance curves for an additional 6 species and 13 populations of Zaprionus. In this data set, populations of $Z$. indianus from both their native and invasive ranges consistently have larger estimates of $B_{50}$ than other populations and species (Figure 8). Two exceptions to this trend are that the $B_{50}$ of $Z$. indianus from Zambia is more similar to $Z$. africanus than other populations of $Z$. indianus, and the $B_{50}$ of $Z$. gabonicus is similar to populations of $Z$. indianus (Figure 8 ). 


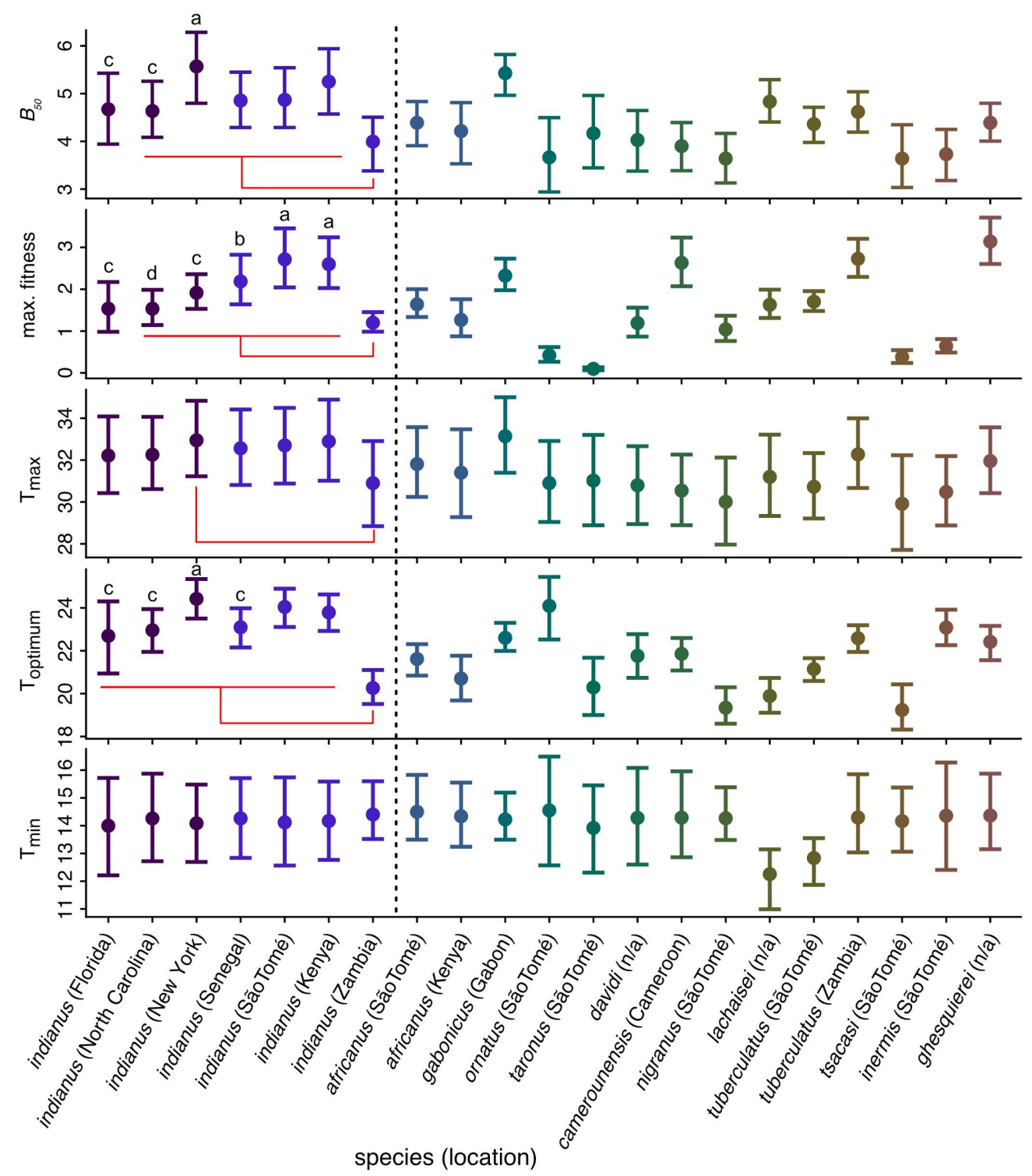

Figure 8. Median and $95 \%$ credible intervals for parameters estimated by jointly fitting performance curves to thermal performance data collected at mean temperatures of 13.9 to $28.9^{\circ} \mathrm{C}$. Populations of $Z$. indianus are shown to the left of the dashed line and all other species of Zaprionus to the right. For populations of Z. indianus, parameter estimates that had a probability greater than 0.95 of being different from one another in pairwise comparisons (analogous to $P<$ 0.05 ) are denoted by letters ('a' > 'c' and 'd', and 'b' > 'd'). Red bars indicate significant differences between the population of $Z$. indianus from Zambia and other populations of $Z$. indianus.

We next tested whether invasive and native populations of $Z$. indianus differed in maximum fitness, as measured by the total number of adult progeny produced by a single pair of flies, at their optimal temperature ("stretch" parameter reported in Tittes et al. 2019; "max. fitness" in Figure 8). African populations of $Z$. indianus from Kenya and São Tomé have higher maximum 
fitness than invasive populations from Florida, New York, and North Carolina, while the population from Senegal only had higher maximum fitness than the invasive population sampled from North Carolina (Figure 8). All populations of $Z$. indianus other than those from Florida and North Carolina had higher maximum fitness than the population from Zambia (Figure 8). The lower maximum fitness observed in invasive populations, compared to populations from Kenya and São Tomé, could be explained by a loss of genetic diversity having negative effects on fertility, fecundity, or survivorship. However, if a loss of genetic diversity underlies a decrease in maximum fitness, one prediction is that these effects would be particularly pronounced at stressful temperatures (i.e. near the minimum and maximum acceptable temperatures), thereby reducing the breadth of the performance curve. This was not the case, as estimates of thermal minima and maxima did not consistently differ between invasive and native populations ( $T_{\min }$ and $T_{\max }$ parameters, respectively; Figure 8). There was also no evidence that maximum fitness in invasive populations (Figure 8) was different from that in the African populations collected in Senegal, despite the latter having high levels of genetic diversity. Therefore, we did not find consistent evidence that the loss of diversity associated with $Z$. indianus's range expansion into North America has reduced the generally high performance that this species displays across a broad range of temperatures.

In addition to comparisons among populations of $Z$. indianus, populations of $Z$. indianus from Kenya and São Tomé both had maximum fitness equal-to or greater than the 12 other species of Zaprionus we measured (Figure 8). The population from Senegal had a similarly high estimate of maximal fitness compared to all other species, except Z. ghesquierei, which showed a higher maximal fitness than all three invasive North American populations of $Z$. indianus, as well as $Z$. indianus from Senegal and Zambia (Figure 8). Three other species were estimated to have a maximal fitness that was greater than $Z$. indianus from at least two of the three North American populations: Z. gabonicus, Z. camerounensis, and Z. tuberculatus. This result suggests that the range of temperatures over which a species or population can maintain relatively high fitness (e.g. $B_{50}$ ) is a more important factor differentiating $Z$. indianus from non-invasive species of Zaprionus than the absolute maximal fitness/performance that individuals in a population can achieve.

\section{Discussion}

Identifying the genetic changes that are associated with biological invasions, along with effects those changes have on performance and fitness, is important for understanding the evolutionary controls acting on invasive species (Lee 2002; Lawson Handley et al. 2011; Stapley et al. 2015; 
Estoup et al. 2016). We have shown that the range expansion of the invasive African fig fly, $Z$. indianus, has resulted in a loss of diversity in invasive populations, but that levels of diversity in invasive populations remain as high or higher than those observed in other species in the genus Zaprionus. The level of genetic diversity we observe in Z. indianus (Figure 1; Table S4) is also similar to those reported for the invasive species Drosophila suzukii (Adrion et al. 2014) and somewhat higher than values that have been reported in populations of $D$. melanogaster (Begun and Aquadro 1992; Langley et al. 2012; Pool et al. 2012). In the case of D. melanogaster, populations in the invasive (or non-native) part of the species' range show geographic and temporal changes in allele frequency that are consistent with adaptation to novel environments (Bergland et al. 2014; Adrion et al. 2015). These interspecific comparisons illustrate how invasive populations with lower genetic diversity than native populations can still have sufficient amounts of genetic diversity to facilitate adaptation to novel environments. Attributes of successful invasive species' biology, such as a generalist life-history (Sakai et al. 2001; Allendorf and Lundquist 2003), can therefore help them maintain genetic diversity and avoid the 'genetic paradox' that may occur under more extreme or stereotyped range expansions (i.e. severe bottleneck with relatively few colonizing individuals).

A number of evolutionary mechanisms that act to increase (or maintain) genetic diversity in invasive populations could also help explain the high levels of genetic diversity in invasive populations of $Z$. indianus. For example, multiple introductions from distinct populations in the native range and admixture can both act to generate high levels of genetic diversity in invasive populations (Stepien et al. 2005; Dlugosch and Hays 2008; Rosenthal et al. 2008; Barker et al. 2017). High levels of genetic diversity in native populations of Z. indianus (Figure 2) may also act to buffer invasive populations against a loss of diversity, but without knowing the source population(s) of invasive $Z$. indianus we are not able to test this hypothesis.

In addition to comparing overall levels of genetic diversity, we were able to use interspecific comparisons to test if and how invasion alters how that genetic diversity is distributed across the genome. Correlated levels of genetic diversity have been shown between a pair of divergent bird species (Dutoit et al. 2017), but to the best of our knowledge, this has not been tested in a phylogenetic context until now. We identified and used the correlation in diversity between species (Figure $5 b$ and $5 c$ ) to show that the genome-wide landscape of genetic diversity is altered in invasive, relative to native, populations of $Z$. indianus (Figure 6 ). We also showed that the difference in the amount of genetic diversity between invasive and native populations of $Z$. 
indianus tends to be larger in regions of the genome with low recombination rates (Figure $3 b$ ). Previous studies have reported a similar pattern with respect to lower genetic diversity on the $X$ chromosome in non-African populations of D. simulans (Begun and Whitley 2000; Schöfl and Schlötterer 2006). We have yet to identify the scaffolds that compose the sex chromosomes in the genome assemblies we report here, but larger reductions in diversity on the $X$-chromosome relative to the autosomes is likely to contribute to the pattern of reduced genetic diversity in regions of low recombination. One mechanism that could explain this pattern is linked selection (Nordborg et al. 1996; Charlesworth et al. 1997; Charlesworth et al. 1997; Begun and Whitley 2000), as selection will have a larger spatial effect on levels of genetic diversity in regions of the genome with low recombination rates. Future work is needed to identify both the chromosome structure of Zaprionus species and regions of the genome potentially subject to selection in invasive populations of $Z$. indianus.

Despite the differences in genetic diversity between invasive and native populations of $Z$. indianus we found that individuals from both regions do not differ in their ability to maintain high performance across a broad range of thermal environments (Figure 8). This finding is in contrast with studies that have found a positive correlation between genetic diversity and measures of fitness ((Reed and Frankham 2003; Leimu et al. 2006; Markert et al. 2010); but see (Lammi et al. 1999)).

The broad thermal niche of $Z$. indianus populations across their native and invasive ranges may help explain this species' high level of genetic diversity and success as an invasive species. For example, if $Z$. indianus evolved a broad thermal niche prior to expanding their range out of Africa, this could have led to large and broadly distributed populations that would build up high levels of genetic diversity. If alleles underlying thermal performance traits became fixed (or present at a high frequency) in native populations prior to range expansion, reductions in genetic diversity would not remove those high-frequency adaptive alleles. This scenario is related to the idea that adaptation to anthropogenically disturbed environments in the native portion of a species' range can facilitate subsequent range expansions (Lee and Gelembiuk 2008; Hufbauer et al. 2012). While the timing of adaptive trait evolution in populations of $Z$. indianus, relative to their range expansion, is not known, anecdotal evidence from collecting Zaprionus across locations in their native Africa suggest that $Z$. indianus possess a generalist and 'invasive-like' life history (Pers. Obs.). For example, we collected $Z$. indianus near cities or rural human settlements in rainforest, savannah, and desert environments, and $Z$. indianus is among the first and most abundant 
species to visit fruit traps across these environments. If the traits that underlie a broad thermal niche evolved in African populations prior to range expansion, then lower diversity in invasive populations may not have negatively affected the fitness of $Z$. indianus in the invasive part of their range.

Estimates of thermal performance curves suggest that, despite the climate being markedly cooler at sample sites in northeastern North America (e.g. North Carolina and New York) than at African sites, populations of $Z$. indianus in North America have not evolved to tolerate more temperate climates that African populations (estimated minimum temperature where fitness falls to 0 does not differ between any population of $Z$. indianus; $T_{\min }$ parameter in Figure 8). Thermal niche breadth also does not systematically differ between Z. indianus from New York, São Tomé and Kenya, and these populations have significantly wider thermal niches than almost all other Zaprionus species or population in our data set (all $P<0.05$ in pairwise comparisons; Figure 9). Together, these results indicate that the thermal niche of North American and African Z. indianus has not evolved to be different, and that the loss of genetic diversity in invasive populations has not reduced the broad range of temperatures across which $Z$. indianus is capable of maintaining relatively high fitness (i.e. $B_{50}$; Figure 8 ).

An alternate interpretation for the lack of differences in thermal performance curves between invasive and native populations of $Z$. indianus is that the lower level of genetic diversity in North American populations of $Z$. indianus is constraining potentially adaptive niche evolution in these populations. Comparing levels of diversity that segregate in Z. indianus to those in other drosophilid populations suggest this is unlikely: invasive populations of $Z$. indianus still harbor as much or more genetic diversity than other species of Zaprionus and Drosophila. However, future work testing levels of additive genetic variation for traits involved in thermal tolerance are needed to quantify the adaptive potential of the different populations and species.

Traits associated with thermal performance are only one suite of traits potentially under selection during colonization and range expansion into novel environments. Other traits such as competitive ability (Blossey and Notzold 1995) and the ability to utilize a broad range of other habitats (Lee and Gelembiuk 2008) can affect the success of invasive species. Of particular note is the fact that we quantified thermal performance on a single food resource (standard cornmeal media for Drosophila). Measuring performance in the same populations and species analyzed here, across a wide range of diets, would be useful to determine the extent to which invasive populations of $Z$. 
indianus have adapted to successfully colonize the broad range of environments they now occupy.

In conclusion, we have shown that genetic diversity in invasive populations of $Z$. indianus is lower than in native populations (Figure 2), and that the genome-wide distribution of genetic variation is perturbed in invasive populations (Figures $3 b, 4,5$, and 6). These results provide a more nuanced understanding of how range expansions associated with invasion can alter levels of genetic variation across the genome. Despite these effects on genetic diversity, invasive populations of $Z$. indianus maintain as much or more genetic diversity than non-invasive congeneric species (Figure 2), and both invasive and native populations of $Z$. indianus are capable of maintaining high fitness across a broad range of thermal environments (Figures 7 and 8). These results show how measures of fitness in invasive species can be decoupled from genetic diversity. They also suggest that adaptation to an "invasive" niche prior to range expansion may be a more important event in the evolutionary history of an invasive lineage than the demographic events that take place during subsequent expansion of their range into novel environments. Future work identifying the evolutionary history of invasive traits (and their underlying genetic variation) will be central to our understanding of the factors controlling the success and spread of invasive species.

\section{Material and Methods}

Detailed methods can be found in the Supplementary Material online.

Population sampling. We sampled wild populations of Drosophilids at five locations across the native range of $Z$. indianus in sub-Saharan Africa and three locations in the invasive range in North America and Hawaii (Figure 1) using traps baited with bananas.

Genome assembly and annotation. To perform population genomic analyses, we first generated genome assemblies de novo for each of the seven Zaprionus species included in this study using data generated from Illumina and Nanopore sequencers. We used the BUSCO annotation pipeline (Waterhouse et al. 2018) to assess assembly quality and quantify the presence of-and generate annotations for-2,799 Benchmarking Universal Single-Copy Orthologs that have been curated in 25 different species of Diptera. Finally, we generated gene annotations using RNA sequence data and the MAKER annotation pipeline (Campbell et al. 2014) for 6 of the 7 de novo genome assemblies (we did not collect RNA-seq data for Z. inermis; SI). Our sequencing, 
assembly, and annotation approaches resulted in genome assemblies with scaffold N50s between $336 \mathrm{~kb}$ and $2.45 \mathrm{Mbp}$, complete single-copy BUSCO annotations of 90.7 to $97.4 \%$, and 9,275 to 11,071 annotated transcripts (Table S2).

Population resequencing and genotyping. To quantify genetic diversity within populations of Zaprionus, we generated resequence data from 93 individuals across 16 populations and 7 species (minimum $\mathrm{N}=3$; maximum $\mathrm{N}=11$; Table $\mathrm{S} 3$ ). We mapped raw sequence reads using the BWA mem algorithm (v0.7.15), sorted and filtered mapped reads using SAMTOOLS (v1.4), marked duplicates using the PICARD MarkDuplicates tool (v2.2.4), and realigned around indels using GATK's RealignerTargetCreator and IndelRealigner tools (v3.8 (McKenna et al. 2010)). We estimated genotypes for each individual using GATK and hard-filtered sites genotyped in fewer than two individuals (VCFtools (v0.1.15) option “--max-missing 0.5”). To facilitate comparisons across populations where we sampled different numbers of individuals, joint genotyping and filtering was carried out on randomly selected groups of four individuals (8 chromosomes) per population, except for the population of $Z$. africanus sampled from São Tomé, where we only sampled three individuals.

Estimating genetic diversity. Population genetic metrics of genetic diversity were computed using VCFtools with coverage-masked sites excluded using the "--exclude-positions" filter option. Because $\pi_{S N P}$ and $S$ were highly correlated in all populations $(r>0.963)$, we focus primarily on $S$ : the number of sites with segregating variation within a given $5 \mathrm{~Kb}$ window.

Comparing genetic diversity within and outside of gene annotations. We used generalized linear models (GLMs) with poisson distributed error $(g \operatorname{lm}()$ function in R) to model the number of segregating sites $(S)$ within a genomic window as a function of the position of that window relative to a gene annotation. For populations of $Z$. indianus, we were also interested in whether aspects of biological invasion had a different effect on levels of genetic diversity depending on the proximity of a genomic region to a gene. We therefore used a GLM to test the interaction between gene region type (i.e. overlapping, adjacent, or distant) and invasion status (i.e. invasive population of $Z$. indianus, native population of $Z$. indianus, or population of non-invasive species of Zaprionus) on median levels of genetic diversity across genomic windows.

Estimating recombination rate and its effect on genetic diversity. To estimate fine-scale population recombination rates $\left(\rho_{\text {rec }}=2 \mathrm{~N} r\right.$ ) across the genome, we used the maximum likelihood method 
implemented in LDhelmet (v1.10; (Chan et al. 2012)). For analyses involving recombination rate, we focused on populations of $Z$. indianus because we had the largest sample size of this species from a single region (Senegal: $N=14$ individuals [28 haplotypes]), which allowed us to use haplotype information to estimate recombination rates across the genome. All analyses below are conducted on mean recombination rates within 5,000 bp genomic windows that were generated from median posterior estimates provided by LDhelmet.

We tested for a relationship between recombination rate and gene density across the 40 largest scaffolds of the $Z$. indianus genome assembly (12,080 windows) using Spearman's rank correlation tests. We found no evidence for a relationship between recombination rate and gene density - either across all windows (Spearman's $\rho=-0.014 ; P=0.12$ ) or windows containing at least one gene ( $\mathrm{N}=4,406$ windows; Spearman's $\rho=0.023 ; P=0.12)$. We therefore conducted independent tests for relationships between genetic diversity and recombination rate and genetic diversity within and around gene annotations.

Measuring the correlation in genetic diversity between species. To facilitate interspecific comparisons, we analyzed genomic regions that spanned the first and last exon of an annotated BUSCO ortholog. In total, we quantified the correlation in diversity (or lack thereof) across 2,714 genomic windows spanning BUSCO orthologs (hereafter "BUSCO windows") annotated across all seven species' genome assemblies. Correlation coefficients were estimated using the rcorr() function from the Hmisc R package. To test whether comparing BUSCO windows produced correlations greater than expected by chance, we performed randomization tests where we randomly selected 2,714 genomic windows (100 iterations) and computed all pairwise Spearman's rank correlation coefficients for those windows across species.

The genomic landscape of diversity may diverge between species with increasing phylogenetic distance due to aspects of genome evolution. To quantify whether this would affect interspecific comparisons of genetic diversity, we computed phylogenetic distances between each species using a concatenated alignment of 1,709 BUSCOs shared across all species genome assemblies and representing 4,616,644 sites. Genetic distances were computed from the identity matrix of the aligned BUSCOs using the dist.alignment() function from the seqinr $\mathrm{R}$ library. We then tested whether the correlation in $S$ across the genome of two species was related to their genetic distance (Spearman's Mantel test; mantel() function in the ecodist R library). We also calculated Spearman's rank correlations for $S$ and Tajima's $D$ between populations of $Z$. indianus (all pairwise comparisons) using diversity estimates across all $5 \mathrm{~kb}$ genomic window. 
Estimating thermal performance curves. To quantify differences in performance across populations, we fit a model that jointly describes the thermal performance curve to data from all populations using a hierarchical Bayesian framework (Tittes et al. 2019). This model provides estimates for the maximum height of a population's performance curve, two shape parameters allowing for asymmetry in the performance curve, and the minimum and maximum temperatures where performance falls to 0 ( $T_{\min }$ and $T_{\max }$ parameters, respectively). The height (i.e. stretch as defined in Tittes et al. 2019) reflects the maximal fitness/performance of a population. We also derived three additional parameters from the five model-estimated parameters to summarize aspects of fitness and the thermal niche: the temperature at which a population displays maximal performance ( $T_{\text {optimum }}$ ), the total area under the estimated performance curve $\left(A_{c}\right)$, and the breadth of the curve between the lower $25 \%$ and the upper $25 \%$ of the curve $\left(B_{50}\right)$. We compared performance curves between species by generating posterior draws of parameter estimates. Two populations were considered to differ with respect to parameters describing their thermal performance curve when a given parameter estimate had a probability greater than 0.95 of being different between the two populations.

\section{Acknowledgements}

We thank M. Cenzer, C. Maxwell, A. Serrato-Capuchina, S. Yeap, E. Behrman, P. Schmidt, B. Cooper, K. Deitz, J. Coughlan, M. Schumer, two anonymous reviewers, and the editor for scientific discussions that improved this manuscript and/or for help in the field. This work was supported by the National Science Foundation (Dimensions of Biodiversity award number 1737752 to D.R.M. and A.H.H.). The funders had no role in an aspect of study design, data collection and analysis, or decisions with respect to publication.

\section{Competing interests}

None of the authors declare any competing interests, financial or otherwise.

\section{References}

Adrion JR, Hahn MW, Cooper BS. 2015. Revisiting classic clines in Drosophila melanogaster in the age of genomics. Trends Genet. TIG 31:434-444.

Adrion JR, Kousathanas A, Pascual M, Burrack HJ, Haddad NM, Bergland AO, Machado H, Sackton TB, Schlenke TA, Watada M, et al. 2014. Drosophila suzukii: the genetic footprint of a recent, worldwide invasion. Mol. Biol. Evol. 31:3148-63.

Agashe D, Bolnick DI. 2010. Intraspecific genetic variation and competition interact to influence niche expansion. Proc. R. Soc. B Biol. Sci. 277:2915-2924. 
Agashe D, Falk JJ, Bolnick DI. 2011. Effects of Founding Genetic Variation on Adaptation to a Novel Resource. Evolution 65:2481-2491.

Aguilar A, Roemer G, Debenham S, Binns M, Garcelon D, Wayne RK. 2004. High MHC diversity maintained by balancing selection in an otherwise genetically monomorphic mammal. Proc. Natl. Acad. Sci. 101:3490-3494.

Allendorf FW, Lundquist LL. 2003. Introduction: Population Biology, Evolution, and Control of Invasive Species. Conserv. Biol. 17:24-30.

Baker HG, Stebbins GL. 1965. The Genetics of Colonizing Species. New York and London: Academic Press

Barker BS, Andonian K, Swope SM, Luster DG, Dlugosch KM. 2017. Population genomic analyses reveal a history of range expansion and trait evolution across the native and invaded range of yellow starthistle (Centaurea solstitialis). Mol. Ecol. 26:1131-1147.

Barker BS, Cocio JE, Anderson SR, Braasch JE, Cang FA, Gillette HD, Dlugosch KM. 2019. Potential limits to the benefits of admixture during biological invasion. Mol. Ecol. 28:100113.

Begun DJ, Aquadro CF. 1992. Levels of naturally occurring DNA polymorphism correlate with recombination rates in $D$. melanogaster. Nature 356:519-520.

Begun DJ, Holloway AK, Stevens K, Hillier LW, Poh Y-P, Hahn MW, Nista PM, Jones CD, Kern $A D$, Dewey CN, et al. 2007. Population genomics: whole-genome analysis of polymorphism and divergence in Drosophila simulans. PLoS Biol. 5:e310.

Bergland AO, Behrman EL, O'Brien KR, Schmidt PS, Petrov DA. 2014. Genomic Evidence of Rapid and Stable Adaptive Oscillations over Seasonal Time Scales in Drosophila. PLoS Genet. 10.

Blossey B, Notzold R. 1995. Evolution of Increased Competitive Ability in Invasive Nonindigenous Plants: A Hypothesis. J. Ecol. 83:887-889.

Burri R, Nater A, Kawakami T, Mugal CF, Olason PI, Smeds L, Suh A, Dutoit L, Bureš S, Garamszegi LZ, et al. 2015. Linked selection and recombination rate variation drive the evolution of the genomic landscape of differentiation across the speciation continuum of Ficedula flycatchers. Genome Res. 25:1656-1665.

Campbell MS, Holt C, Moore B, Yandell M. 2014. Genome Annotation and Curation Using MAKER and MAKER-P. Curr. Protoc. Bioinforma. 48:4.11.1-39.

Chan AH, Jenkins PA, Song YS. 2012. Genome-Wide Fine-Scale Recombination Rate Variation in Drosophila melanogaster. PLOS Genet. 8:e1003090.

Charlesworth B, Nordborg M, Charlesworth D. 1997. The effects of local selection, balanced polymorphism and background selection on equilibrium patterns of genetic diversity in subdivided populations. Genet. Res. 70:155-174.

Charlesworth D. 2006. Balancing Selection and Its Effects on Sequences in Nearby Genome Regions. PLOS Genet. 2:e64.

Dlugosch KM, Anderson SR, Braasch J, Cang FA, Gillette HD. 2015. The devil is in the details: genetic variation in introduced populations and its contributions to invasion. Mol. Ecol. 24:2095-2111.

Dlugosch KM, Hays CG. 2008. Genotypes on the move: some things old and some things new shape the genetics of colonization during species invasions. Mol. Ecol. 17:4583-4585.

Dlugosch KM, Parker IM. 2008. Founding events in species invasions: genetic variation, adaptive evolution, and the role of multiple introductions. Mol. Ecol. 17:431-449.

Dutoit L, Vijay N, Mugal CF, Bossu CM, Burri R, Wolf J, Ellegren H. 2017. Covariation in levels of nucleotide diversity in homologous regions of the avian genome long after completion of lineage sorting. Proc. R. Soc. B Biol. Sci. 284:20162756.

Ellegren H, Galtier N. 2016. Determinants of genetic diversity. Nat. Rev. Genet. 17:422-433.

Ellstrand NC, Schierenbeck KA. 2000. Hybridization as a stimulus for the evolution of invasiveness in plants? Proc. Natl. Acad. Sci. 97:7043-7050. 
Estoup A, Ravigné V, Hufbauer R, Vitalis R, Gautier M, Facon B. 2016. Is There a Genetic Paradox of Biological Invasion? Annu. Rev. Ecol. Evol. Syst. 47:51-72.

Facon B, Pointier J-P, Jarne P, Sarda V, David P. 2008. High Genetic Variance in Life-History Strategies within Invasive Populations by Way of Multiple Introductions. Curr. Biol. 18:363-367.

Gibert P, Hill M, Pascual M, Plantamp C, Terblanche JS, Yassin A, Sgr?? CM. 2016. Drosophila as models to understand the adaptive process during invasion. Biol. Invasions 18:10891103.

Gillespie JH. 1994. Substitution processes in molecular evolution. III. Deleterious alleles. Genetics 138:943-952.

Grapputo A, Boman S, Lindström L, Lyytinen A, Mappes J. 2005. The voyage of an invasive species across continents: genetic diversity of North American and European Colorado potato beetle populations. Mol. Ecol. 14:4207-4219.

Hovick SM, Whitney KD. 2014. Hybridisation is associated with increased fecundity and size in invasive taxa: meta-analytic support for the hybridisation-invasion hypothesis. Ecol. Lett. 17:1464-1477.

Hufbauer RA, Facon B, Ravigné V, Turgeon J, Foucaud J, Lee CE, Rey O, Estoup A. 2012. Anthropogenically induced adaptation to invade (AIAI): contemporary adaptation to human-altered habitats within the native range can promote invasions. Evol. Appl. 5:89101.

Hyten DL, Song Q, Zhu Y, Choi I-Y, Nelson RL, Costa JM, Specht JE, Shoemaker RC, Cregan PB. 2006. Impacts of genetic bottlenecks on soybean genome diversity. Proc. Natl. Acad. Sci. 103:16666-16671.

Kolbe JJ, Glor RE, Rodríguez Schettino L, Lara AC, Larson A, Losos JB. 2004. Genetic variation increases during biological invasion by a Cuban lizard. Nature 431:177-181.

Langley $\mathrm{CH}$, Stevens K, Cardeno C, Lee YCG, Schrider DR, Pool JE, Langley SA, Suarez C, Corbett-Detig RB, Kolaczkowski B, et al. 2012. Genomic Variation in Natural Populations of Drosophila melanogaster. Genetics 192:533-598.

Lavergne S, Molofsky J. 2007. Increased genetic variation and evolutionary potential drive the success of an invasive grass. Proc. Natl. Acad. Sci. 104:3883-3888.

Lawson Handley LJ, Estoup A, Evans DM, Thomas CE, Lombaert E, Facon B, Aebi A, Roy HE. 2011. Ecological genetics of invasive alien species. BioControl 56:409-428.

Lee CE. 2002. Evolutionary genetics of invasive species. Trends Ecol. Evol. 17:386-391.

Lee CE, Gelembiuk GW. 2008. Evolutionary origins of invasive populations. Evol. Appl. 1:427448.

Lindtke D, Lucek K, Soria-Carrasco V, Villoutreix R, Farkas TE, Riesch R, Dennis SR, Gompert Z, Nosil P. 2017. Long-term balancing selection on chromosomal variants associated with crypsis in a stick insect. Mol. Ecol. 26:6189-6205

Manichaikul A, Mychaleckyj JC, Rich SS, Daly K, Sale M, Chen W-M. 2010. Robust relationship inference in genome-wide association studies. Bioinformatics 26:2867-2873.

Markert JA, Champlin DM, Gutjahr-Gobell R, Grear JS, Kuhn A, McGreevy TJ, Roth A, Bagley MJ, Nacci DE. 2010. Population genetic diversity and fitness in multiple environments. BMC Evol. Biol. 10:205.

Marsden CD, Vecchyo DO-D, O'Brien DP, Taylor JF, Ramirez O, Vilà C, Marques-Bonet T, Schnabel RD, Wayne RK, Lohmueller KE. 2016. Bottlenecks and selective sweeps during domestication have increased deleterious genetic variation in dogs. Proc. Natl. Acad. Sci. 113:152-157.

Michaelides SN, Goodman RM, Crombie RI, Kolbe JJ. 2018. Independent introductions and sequential founder events shape genetic differentiation and diversity of the invasive green anole (Anolis carolinensis) on Pacific Islands.Cowie R, editor. Divers. Distrib. 24:666-679. 
Nachman MW. 2001. Single nucleotide polymorphisms and recombination rate in humans. Trends Genet. 17:481-485.

Oakley CG, Lundemo S, Ågren J, Schemske DW. 2019. Heterosis is common and inbreeding depression absent in natural populations of Arabidopsis thaliana. J. Evol. Biol. 32:592603.

Otto SP, Whitlock MC. 1997. The Probability of Fixation in Populations of Changing Size. Genetics 146:723-733.

Pool JE, Corbett-Detig RB, Sugino RP, Stevens KA, Cardeno CM, Crepeau MW, Duchen P, Emerson JJ, Saelao P, Begun DJ, et al. 2012. Population Genomics of Sub-Saharan Drosophila melanogaster: African Diversity and Non-African Admixture. PLOS Genet. 8:e1003080.

Pryszcz LP, Gabaldón T. 2016. Redundans: an assembly pipeline for highly heterozygous genomes. Nucleic Acids Res. 44:e113-e113.

Rogers RL, Slatkin M. 2017. Excess of genomic defects in a woolly mammoth on Wrangel island. PLOS Genet. 13:e1006601.

Rosenthal DM, Ramakrishnan AP, Cruzan MB. 2008. Evidence for multiple sources of invasion and intraspecific hybridization in Brachypodium sylvaticum (Hudson) Beauv. in North America. Mol. Ecol. 17:4657-4669.

Sakai AK, Allendorf FW, Holt JS, Lodge DM, Molofsky J, With KA, Baughman S, Cabin RJ, Cohen JE, Ellstrand NC, et al. 2001. The Population Biology of Invasive Species. Ann. Rev. Ecol. Syst. 32:305-332.

Samuk K, Owens GL, Delmore KE, Miller SE, Rennison DJ, Schluter D. 2017. Gene flow and selection interact to promote adaptive divergence in regions of low recombination. Mol. Ecol. 26:4378-4390.

Stapley J, Santure AW, Dennis SR. 2015. Transposable elements as agents of rapid adaptation may explain the genetic paradox of invasive species. Mol. Ecol. 24:2241-2252.

Stepien CA, Brown JE, Neilson ME, Tumeo MA. 2005. Genetic Diversity of Invasive Species in the Great Lakes Versus Their Eurasian Source Populations: Insights for Risk Analysis. Risk Anal. 25:1043-1060.

Tittes SB, Walker JF, Torres-Martínez L, Emery NC. 2019. Grow Where You Thrive, or Where Only You Can Survive? An Analysis of Performance Curve Evolution in a Clade with Diverse Habitat Affinities. Am. Nat::000-000.

Tsutsui ND, Suarez AV, Holway DA, Case TJ. 2000. Reduced genetic variation and the success of an invasive species. Proc. Natl. Acad. Sci. U. S. A. 97:5948-5953.

Waterhouse RM, Seppey M, Simão FA, Manni M, loannidis P, Klioutchnikov G, Kriventseva EV, Zdobnov EM. 2018. BUSCO Applications from Quality Assessments to Gene Prediction and Phylogenomics. Mol. Biol. Evol. 35:543-548. 